mutations). RTK-RAS and TP53 signalling pathways were also found to be altered in both proximal and distal CRCs with six mutations in both pathways. TGF-Beta signalling (four mutations) and PI3K signalling (two mutations) pathways were only altered in our proximal CRCs. There were more altered pathways in proximal as compared to distal CRCs but the difference was not significant $(p=0.66)$.

Conclusions We found that proximal CRCs were presented with more variants and involved different pathways as compared to distal CRCs. Our findings suggest different pathways to tumourigenesis in proximal and distal CRCs that may be the cause of clinical differences. Further study in larger series of samples coupled with functional studies will be needed to confirm the identified variants and determine their role in the genesis of proximal and distal CRCs.

\section{IDDF2018-ABS-0227 SACCHAROMYCES POMBE MAY BE A DOUBLE-EDGE SWORD IN THE GUTS OF MALAYSIAN ADULTS: AN EVIDENCE FROM THE GUT MICROBIOME SECRETOME STUDY}

${ }^{1}$ Siok-Fong Chin*, ${ }^{1}$ Putri Intan Hafizah Megat Mohd Azlan, ${ }^{2}$ Luqman Mazlan, ${ }^{1}$ Huimin Neoh, ${ }^{1}$ Rahman Jamal. ${ }^{1}$ UKM Medical Molecular Biology Institute, Universiti Kebangsaan Malaysia, Cheras, Kuala Lumpur, Malaysia; ${ }^{2}$ Department of Surgery, Faculty of Medicine, Universiti Kebangsaan Malaysia Medical Centre, Cheras, Kuala Lumpur, Malaysia

\subsection{6/gutjnl-2018-IDDFabstracts.31}

Background Dysbiosis of the gut microbiome has been postulated as the causative event of colorectal cancer (CRC). In recent years, consumption of certain strain of microbes in pursuit of correcting gut dysbiosis has been hyped up by the probiotics industry. However, little is known regarding the contribution of these naturally occurring microbes to the gut microenvironment and their pathophysiology interaction with the host in health and diseases, particularly at the molecular level in a chronic term. Therefore, we aim to identify the secreted proteins released from the human gut microflora by assessing the secretome in the stool samples.

Methods Stools samples from 26 clinically-diagnosed patients with CRC and 20 non-CRC control individuals were collected, homogenised and filtered followed by protein extraction and profiling by quantitative label-free proteomics using Nano-Liquid Chromatography TripleTOF Mass Spectrometry. The mass spectra datasets were searched using MaxQuant against the microbial Uniprot Fasta database. Statistical analyses were performed using Mann-Whitney, Kruskal-Wallis and Spearman correlation with $p$-value less than 0.05 .

Results We have identified a total of 649 proteins secreted by the gut flora (253 from yeasts; 404 from bacteria) with 35 proteins specific to CRC, whereas 613 proteins were exclusive to the non-CRC control. Only one yeast protein was found to be secreted in both CRC and non-CRC groups. Interestingly, most significant proteins that were expressed independently in CRC and non-CRC were proteins secreted by Saccharomyces pombe $(\mathrm{p}<0.05)$, distinguished only by its type. Saccharomyces pombe have released a totally different set of significant proteins into the CRC gut (deoxycytidylate deaminase, VMS1 homolog C1827.04, conserved oligomeric Golgi complex subunit 8 and DNA repair protein rhp57) which are also found to be specific to the staging of the disease.
Conclusions Saccharomyces pombe may play a major role in the human gut by distinguishing the CRC-stricken gut from the non-CRC, as was shown by the different set of proteins being released. However, it is still unknown whether the differences found in this study was the cause or effect of dysbiosis that eventually leads to CRC, thus warrants further investigation.

\section{IDDF2018-ABS-0228 HUMAN GUT SECRETOME PROFILING IN MALAYSIAN ADULTS: A CASE STUDY ON COLORECTAL CANCER}

${ }^{1}$ Putri Intan Hafizah Megat Mohd Azlan*, 'Siok-Fong Chin, ${ }^{2}$ Luqman Mazlan, ${ }^{1}$ Huimin Neoh, ${ }^{1}$ Rahman Jamal. ${ }^{1}$ UKM Medical Molecular Biology Institute Universiti Kebangsaan Malaysia, Cheras, Kuala Lumpur, Malaysia; ${ }^{2}$ Department of Surgery, Faculty of Medicine, Universiti Kebangsaan Malaysia Medical Centre, Cheras, Kuala Lumpur, Malaysia

\subsection{6/gutjn|-2018-IDDFabstracts.32}

Background Over the recent years, there has been a paradigm shift in the landscape of understanding colorectal cancer (CRC). Secretomics, a subset of oncoproteomics has emerged as an important tool for CRC biomarker discovery as tumours are known to secrete carcinogenic factors essential for cancer pathogenesis. However, secretomics studies in CRC had mainly focused on in-vitro human cell lines instead of direct assessment from the human gut. Therefore, we aim to identify the secreted proteins released from the human CRC-stricken gut by assessing the secretome in the stool samples.

Methods Stools samples from 26 clinically-diagnosed CRC patients and 20 non-CRC control individuals were collected, homogenised and filtered followed by protein extraction and profiling by quantitative label-free proteomics using NanoLiquid Chromatography TripleTOF Mass Spectrometry. The mass spectra datasets were searched using MaxQuant against the Homo sapiens Uniprot Fasta database. Statistical analyses were performed using Mann-Whitney, Kruskal-Wallis and Spearman correlation with $p$-value $<0.05$.

Results We identified a total of 3565 proteins secreted by human gut with 29\% (1045 proteins) of the proteins, exclusively expressed in CRC and 64.4\% (2296 proteins) in nonCRC. Intriguingly, out of the $1045 \mathrm{CRC}$-associated proteins, we found 12 gender-specific proteins that were significantly secreted in the female patients $(p<0.05)$. Another 120 CRCexclusive proteins were identified from the Indian descent $(\mathrm{p}<0.05)$. Most importantly, we also observed two significant proteins; cancer/testis antigen $47 \mathrm{~B}$ and haptoglobin that were exclusively expressed in Stage I and Stage IV CRC $(\mathrm{p}<0.05)$, respectively. As for the non-CRC guts, we have found 11 proteins adversely correlated with advancing age $(\mathrm{p}<0.05)$. These proteins were previously associated with tumour suppressor role suggesting possible protective mechanisms conferred by the proteins.

Conclusions Proteins identified in this secretome study of CRC-stricken gut have been previously associated with CRC carcinogenesis and were reported in tissues and serum samples. We are the first group to report such proteins as being secreted by human gut and detectable in the stool. Taken together, these proteins could be viewed as a promising biomarker for CRC diagnosis or prognosis. 